\title{
Comparison of the efficiency of colorectal cancer screening programs based on age and genetic risk for reduction of colorectal cancer mortality
}

\begin{abstract}
Oliver Stanesby ${ }^{\star, 1,2}$ and Mark Jenkins ${ }^{1}$
Given that colorectal cancer risk depends partly on inherited factors, screening program efficiency may be increased by incorporating genetic factors. We compared the efficiency of screening based on age and genetic risk in a simulated population. We simulated a population matching the size, age distribution and colorectal cancer incidence and mortality of Australia. We also simulated the distribution of genetic risk for colorectal cancer based on the expected number of inherited risk alleles of 45 single-nucleotide polymorphisms (SNPs) previously reported as associated with colorectal cancer. We compared the expected colorectal cancer deaths under three screening programs; age-based, genetic-based and combined age-based and genetic-based. The age-based program would prevent 25.4 deaths per 100000 invited to screen, none of which would be under age 50; the genetic program would prevent 26.2 deaths per 100000 invited to screen, 16 of which would be under age 50; and the combined program would prevent 24.4 deaths per 100000 invited to screen, 16 of which would be under age 50. Genetic testing of 1.5 million $45-49$ year olds would identify $91 \%$ of the people aged under 50 at sufficient risk to warrant screening, potentially saving 16 colorectal cancer deaths each year. Screening eligibility based on genetic risk profile for age is as efficient as eligibility based on age alone for preventing colorectal cancer mortality, but identifies an additional $7 \%$ of the population at sufficient risk to benefit from screening who would not normally be screened given they are aged under 50 years.
\end{abstract}

European Journal of Human Genetics (2017) 25, 832-838; doi:10.1038/ejhg.2017.60; published online 10 May 2017

\section{INTRODUCTION}

Colorectal cancer screening aims to reduce colorectal cancer incidence and mortality through prevention and early identification of disease. ${ }^{1,2}$ Given that age is the strongest risk factor for the disease (an estimated $93 \%$ of colorectal cancers are diagnosed and $95 \%$ of colorectal cancer mortalities occur, in people aged 50 years and above ${ }^{3}$ ), many countries either offer, or are building to offer, free biennial immunochemical fecal occult blood test (iFOBT) screening to those within a specific age group, usually from age 50 to 74 years. ${ }^{4}$ However, given that most people do not develop colorectal cancer and there is a wide risk of colorectal cancer across the population, an age-based program is not optimal. Although the majority of colorectal cancers are diagnosed within the 50-74-year-old age group, the vast majority (>90\%) of people in this age range will not develop colorectal cancer and a proportion of those under 50 will develop colorectal cancer.

It has long been known that people with a family history of colorectal cancer, are on average twice as likely as those without any family history to develop colorectal cancer, ${ }^{5}$ which is indicative of genetic risk factors. Several genes have been identified, which when inherited in a mutated form, substantially increase the risk of colorectal cancer (eg, the mismatch repair genes, APC and MUTYH); although these mutation carriers are rare.

Recently, another category of genetic risk factors has been discovered, which are common enough to be relevant to large sections of the population. Single-nucleotide polymorphisms (SNPs), also known as common genetic susceptibility loci, are genetic variants with common population frequency. At least 45 SNPs are associated with risk of colorectal cancer. ${ }^{6}$ Independently, these SNPs are only weakly associated with colorectal cancer risk, but in combination can be used to stratify the population into risk categories for screening. ${ }^{6-9}$ Therefore, genetic risk profiling may improve the efficiency of colorectal cancer screening programs.

The advancement in genetic research and technology ensures it will soon be possible to provide a relatively cheap, quick and accurate assessment of individual risk of colorectal cancer based on their inherited genetic code. ${ }^{10}$ Furthermore, while the cumulative cost of genetic profiling the population is a barrier, ${ }^{11}$ to estimate genetic risk requires a once-off genetic test, rather than repeated measurement, which is advantageous to the feasibility of population-level risk stratification. In combination with non-genetic and lifestyle risk factors, risk models that include SNPs may improve discriminatory accuracy $^{7}$ and efficiency of familiarly associated oncological disease screening programs $s^{9,12,13}$ - although potentially at the expense of sensitivity ${ }^{9,11}$ - and when considered in conjunction with age, can identify a proportion of the population aged under 60 years whose absolute risk of developing colorectal cancer is equal to or greater than the average 60-year-old. ${ }^{9}$ Whether screening programs should exclude people of eligible age from screening on the basis of low genetic risk warrants ethical debate. ${ }^{11}$ However, colorectal cancer screening programs may benefit if population genetic risk can identify

${ }^{1}$ Centre for Epidemiology and Biostatistics, Melbourne School of Population and Global Health, University of Melbourne, Melbourne, VIC, Australia; ${ }^{2}$ Centre for Alcohol Policy Research, Department of Public Health, La Trobe University, Melbourne, VIC, Australia

*Correspondence: O Stanesby, Centre for Alcohol Policy Research, Department of Public Health, La Trobe University, 215 Franklin Street, Melbourne, VIC 3000, Australia. Tel: +613 9479 8733; E-mail: 0.stanesby@latrobe.edu.au

Received 15 September 2016; revised 5 March 2017; accepted 28 March 2017; published online 10 May 2017 
individuals who are ineligible for screening based on age, but who have sufficient predicted risk to benefit from screening.

Here, we assess the potential utility of colorectal cancer screening based on age and SNPs to prevent deaths from colorectal cancer.

\section{MATERIALS AND METHODS}

\section{Data sources and sample population}

Our study sample was a hypothetical population with the same age and sex structure, colorectal cancer incidence and colorectal cancer mortality as the Australian population on 30 June $2011 .^{3}$ We obtained age- and sex-specific population counts, and colorectal cancer incidence and mortality rates for 5 -year age bands $(0-4,5-9 \ldots 80-84, \geq 85$ years). From these data, we derived single-year age bands by dividing the population frequency of each 5 -year ageband by five.

Colorectal cancer was defined as malignant neoplasms of the colon, rectosigmoidal junction or rectum. Colorectal cancer mortalities included all deaths in which colorectal cancer was reported as the underlying cause of death.

To calculate the population distribution of risk based on age and SNPs, we used the data set and analysis of a previous simulation study ${ }^{6}$ in which one million cases of colorectal cancer and one million controls were simulated with the distribution of risk alleles of 45 colorectal cancer SNPs based on the published risk allele frequencies and per-risk allele odds ratios for colorectal cancer (Supplementary Figure 1). To ascertain the independent contribution of each SNP to colorectal cancer risk, the authors excluded SNPs that had sufficient evidence that their association with colorectal cancer was due to linkage disequilibrium with another SNP in the same region (indicated by a lack of statistically significant association with risk of colorectal cancer when included in a multivariate logistic regression model, which contained other SNPs within the same region, or a D prime $>0.5$ with other SNPs within the same region). ${ }^{6} \mathrm{~A}$ description of the $45 \mathrm{SNPs}$ reported to be independently associated with colorectal cancer, including nomenclature, location, per-allele odds ratio and risk allele frequency, is provided in Supplementary Table 1, and further information is available via the primary genome-wide association studies from which associations are estimated.

\section{Screening program eligibility threshold calculations}

The hypothetical population was considered static in terms of age distribution and screening was assumed to comprise biennial iFOBT. Eligibility for screening for each of the three programs considered were: age-based program - all those aged between 50 and 74 years inclusive; genetics-based program - all those with a 5-year risk of colorectal cancer (based on their age and their SNP risk) that surpassed the 5-year risk of being diagnosed with colorectal cancer for Australians aged 50 years in 2011; and integrated program - all those eligible for the age-based program or the genetics-based program. We used the following formula to calculate the 5-year risk of colorectal cancer diagnosis according to age:

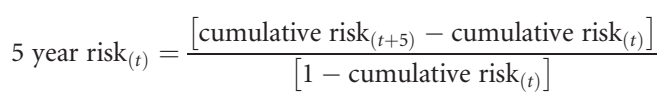

where $t$ is age (years).

We used the following formula to calculate absolute 5-year risk of colorectal cancer diagnosis according to age and SNPs risk:

$$
5 \text { year } \operatorname{risk}_{(t x)}=5 \text { year } \operatorname{risk}_{(t)} \times\left[\frac{\text { odds of colorectal cancer } \mid(x)}{\text { odds of colorectal cancer } \mid(x=40)}\right]
$$

where $t$ is age (years) and $x$ is the number of risk alleles (population mean $=40$ ).

We calculated the 5-year risk of colorectal cancer diagnosis for all possible combinations of number of inherited risk alleles of the 45 SNPs that were previously simulated in the one million controls ${ }^{6}$ (range $=26$ risk alleles to 57 risk alleles) at all variations of age (range $=0$ years to $\geq 85$ years; Supplementary Figure 2). For each age group (year), we established the minimum number of inherited risk alleles required to surpass the eligibility threshold for screening.

\section{Estimating screening program efficiency}

To compare screening program efficiency, we estimated for our hypothetical population under each screening program: the number of people that would be eligible for biennial iFOBT screening, the number of people that would be expected to participate in biennial screening assuming age-specific participation rates (based on the most recent data from the Australian National Bowel Cancer Screening Program - Supplementary Table 2), the expected number of colorectal cancer deaths and years of life lost (YLL) in the screening-eligible population given no screening, and deaths and YLLs expected to be prevented by screening assuming 100\% participation and assuming age-specific participation rates (Supplementary Table 2). For the age-based program, we estimated the number of people that would be eligible for screening every 2 years as the sum of the population frequencies in age groups ranging from 50 to 74 years. For the genetics-based program, we estimated the number of people that would be eligible for screening every 2 years as the sum of individuals who inherited at least the minimum number of risk alleles to surpass the eligibility threshold according to their age (Supplementary Figure 2).

We estimated the expected deaths due to colorectal cancer that would have occurred in a single year in the age-based program's eligible population as the sum of individuals eligible for screening in each age group (years) multiplied by the corresponding age-specific crude rate of deaths due to colorectal cancer per 100000 persons during 2011. We estimated the expected deaths due to colorectal cancer that would have occurred in the genetics-based programeligible population as the sum of expected deaths due to colorectal cancer in all eligible age groups (years) multiplied by the mean odds ratio for colorectal cancer of each eligible age group (years) based on the number of risk alleles. Mean odds ratio for colorectal cancer of each eligible age group (year) was calculated as:

$$
O R_{\text {average }(t)}=\mathrm{e}^{\frac{\sum_{i}\left[\ln \left(O R_{(x)}\right) \times N_{(x)}\right]}{\sum_{i}^{N}(x)}}
$$

where $t$ is age (years), $x$ is the number of risk alleles, $i$ is all eligible variations of $x$ for $t$ and $N$ is the population size for $i$.

We estimated the expected YLLs that would have occurred for each age-year by multiplying expected deaths of each age group (year) by the corresponding Australian age-specific remaining life expectancy. ${ }^{14}$ These were summed to estimate the expected YLLs that would have occurred in the screening-eligible population for each screening program.

We calculated the estimated number of deaths due to colorectal cancer that would be prevented by the screening programs by multiplying expected deaths due to colorectal cancer in the eligible population per year, by the estimated mortality rate reduction of $16 \%$ under expected participation (Supplementary Table 2), and by $44 \%$ when assuming $100 \%$ screening participation. These estimates were based on an observed biennial iFOBT screening program, which reduced colorectal cancer mortality rate by $22 \%$ with a $50 \%$ screening participation. ${ }^{15}$ Similarly, we estimated the YLLs that would be prevented by multiplying expected YLLs due to colorectal cancer in the eligible population of each screening program during 2011 given no screening, by the estimated mortality rate reduction of $16 \%$ when applying age-specific screening participation rates, and $44 \%$ when assuming $100 \%$ screening participation.

We estimated the number of people that would be eligible to screen under the genetics-based program, but not eligible under the age-based program as all people aged less than 50 years whose combined risk surpasses the screening threshold. We estimated the number of people that would be eligible under the age-based program, but not eligible under the genetics-based program as all people aged between 50 and 74 years whose combined age and genetic risk is less than the screening threshold.

We estimated the number of false-positive screening test results, under the assumption that iFOBT has a positive predictive value of $3.6 \%,{ }^{16}$ using the following formula:

$$
N, \text { false positives }=\left[\frac{N, \text { true positives }}{\text { positive predictive value }}\right]-N \text {, true positives }
$$

From this, we estimated the number of serious complications due to follow-up colonoscopy procedures in people who received a false-positive screening result (ie, unnecessary colonoscopies), under the assumption that $1.6 \%$ of 
colonoscopy procedures result in an unplanned hospital visit due to serious complications (including hemorrhage, abdominal pain and perforation). ${ }^{17}$

All the calculations and analyses were completed in Microsoft Excel version 2013

\section{RESULTS}

\section{Characteristics of the hypothetical population}

The population comprised 22340024 people ranging in age from 0 to 85 or greater years with a median age of 37 years, and interquartile range of 36 years $(\mathrm{IQR}=19,55)$. The crude incidence of colorectal cancer in a single year was 67.8 per 100000 persons, and the crude colorectal cancer death rate was 17.8 per 100000 persons.

A person aged 50 years would have an estimated 5-year colorectal cancer risk of $0.33 \%$ and this value was used as the threshold colorectal cancer risk for screening, ie, people were considered eligible to screen under the genetics-based program or the integrated program if their 5-year colorectal cancer risk due to their combined SNPs and age was equal to or greater than $0.33 \%$. The minimum number of inherited risk alleles required to surpass the genetics-based screening program eligibility for each age group are presented in Supplementary Figure 2.

\section{Screening program characteristics: eligible and participating populations}

The eligible and participating number of people in the hypothetical population of the age-based and genetics-based screening programs are detailed in Table 1, Figure 1 and Supplementary Table 3. Under the age-based program, 2868994 people would be invited to screen between the ages of 50 and 74; and under the genetics-based program, 192286 people aged under 50 years and 2513817 people aged 50-74 years would be invited to screen (total invited to screen $=2706103$ ). Under the integrated program, 3061280 would be eligible to screen. The proportion of each age group under age 50, who had a 5 -year risk of colorectal cancer of $0.33 \%$ or higher increased from $0.25 \%$ at age 40 ( 1 in 400 ) to $8.4 \%$ at age 45 ( 1 in 12 ) to $33 \%$ at age 49 (one in three). For 45-49 year-olds the proportion exceeding the threshold risk for screening would be $23 \%$ (one in four; Supplementary Table 3). Compared with the age-based program, 355177 fewer people aged 50 or over would be eligible to screen under the genetics-based program as their risk was less than the screening threshold risk for screening.

\section{Screening program efficiency}

In a single year, the genetics-based screening program would have prevented an estimated six fewer colorectal cancer deaths (deaths prevented $=261$ ), compared with the age-based program (deaths prevented $=267$ ). These prevented deaths would have equated to 63 fewer YLLs prevented by the genetics-based screening program (YLLs prevented $=5515$ ), compared with the age-based program (YLLs prevented $=5578$ ).

The genetics-based program would have prevented more deaths and YLLs per 100000 eligible persons, and would have required less eligible people to be screened to prevent deaths and YLLs (Table 1). Under the genetics-based program and assuming 100\% participation in screening, 409 fewer people would have needed to be invited to screen to prevent one colorectal cancer death $(\mathrm{NNiS}=10354)$, and 25 fewer people would have needed to be invited to screen to prevent one YLL (NNiS $=490$ ), compared with the age-based program (NNiS to prevent one colorectal cancer death $=10763$; NNiS to prevent one YLL =515). Taking participation rates into consideration, the genetics-based program would have required 120 fewer people to participate in the screening to prevent one death $(\mathrm{NNS}=3811)$, and seven fewer people to participate in the screening to prevent one YLL (NNS $=181$ ), compared with the age-based program (NNS to prevent one death $=3931$; NNS to prevent one YLL $=188$ ). In a single year, the genetics-based program would have also resulted in 759 fewer false-positive screening test results, and therefore 12 fewer serious complications due to undergoing unnecessary colonoscopy compared with the age-based program.

A total of 192286 people would be eligible to screen under the genetics-based program, but not eligible under the age-based program (age range $=32$ years, 49 years; Figure 1). By inviting people aged under 50 years with at least a $0.33 \% 5$-year risk of colorectal cancer to screen, the genetics-based program would have prevented six colorectal cancer deaths, equating to 222 YLLs in a single year that would not have been prevented by the age-based program. A total of 355177 people would be eligible under the age-based program, but not under the genetics-based program (range $=50$ years, 74 years). By not inviting people aged 50 years and above with less than a $0.33 \% 5$ year risk of colorectal cancer, the genetics-based program would have failed to prevent seven colorectal cancer deaths, equating to 214 YLLs that would be preventable under the age-based program.

\section{Combining age-based and genetics-based screening programs: an integrated approach}

The eligible and participating people of the integrated program are detailed in Table 1 and Figure 1. Compared with the age-based program, 192286 more people would be eligible to screen under the integrated program $(N=3061280$; range $=32$ years, 74 years $)$.

In a single year, the integrated screening program would have prevented an estimated five more colorectal cancer deaths (deaths prevented $=272$ ), and 222 more YLLs (YLLs prevented $=5800$ ), but 729 more false-positive screening results and 12 more serious complications due to subsequent colonoscopy, compared with the age-based program. Under the integrated program, the number needed to invite to screen was 466 greater to prevent one death $(\mathrm{NNiS}=11229)$, and 13 greater to prevent one YLL $(\mathrm{NNiS}=466)$, compared with the age-based program. Furthermore, the number needed to participate in screening was 105 greater to prevent one death (NNS =4036), and two greater to prevent one YLL $(\mathrm{NNS}=190)$, compared with the age-based program.

\section{DISCUSSION}

We have demonstrated that eligibility for colorectal cancer screening (biennial FOBT) based on genetic risk for age is slightly more efficient in terms of colorectal cancer deaths and reduction in years of life lost per person invited to screen, compared with eligibility based on being aged between 50 and 74 years. For a population the size and age distribution of Australia in 2011, the genetics-based program would have invited approximately 192000 people under age 50 who would not be eligible for age-based screening, yet had a risk of colorectal cancer at least as high as the average 50-year-old. The genetics-based program would also have resulted in approximately 355000 people between 50 and 74 being classified as no longer eligible for screening as they had a risk of colorectal cancer less than the average 50-yearold. We estimated that the net effect of the genetics-based program would be $0.4 \%$ more colorectal cancer deaths $(n=6)$ and $0.2 \%$ more YLLs $(n=63)$ than the age-based program, but $4.9 \%$ fewer screens $(n=51548)$ resulting in a $3.1 \%$ improved efficiency (screens per death). If the genetics program was deployed in addition to the agebased program (the integrated program), there would be $0.3 \%$ fewer deaths than the age-based program $(n=5)$ but $5.0 \%$ more screens 
Table 1 For three colorectal cancer screening programs (age, genetics and integrated) conducted in a theoretical population resembling the size and age distribution of Australia in 2011 ( $N=22340$ 042): the number of people eligible for biennial screening, number of people invited to screen each year, number of people expected to participate, number of expected colorectal cancer deaths and years of life lost to colorectal cancer (YLL) if there was no screening, the number of colorectal cancer deaths and YLL prevented by biennial screening, and the number of false positive screening tests and serious consequences of false positive screening tests in a single year assuming age-specific participation rates, and assuming all eligible persons participate in screening ( $100 \%$ participation)

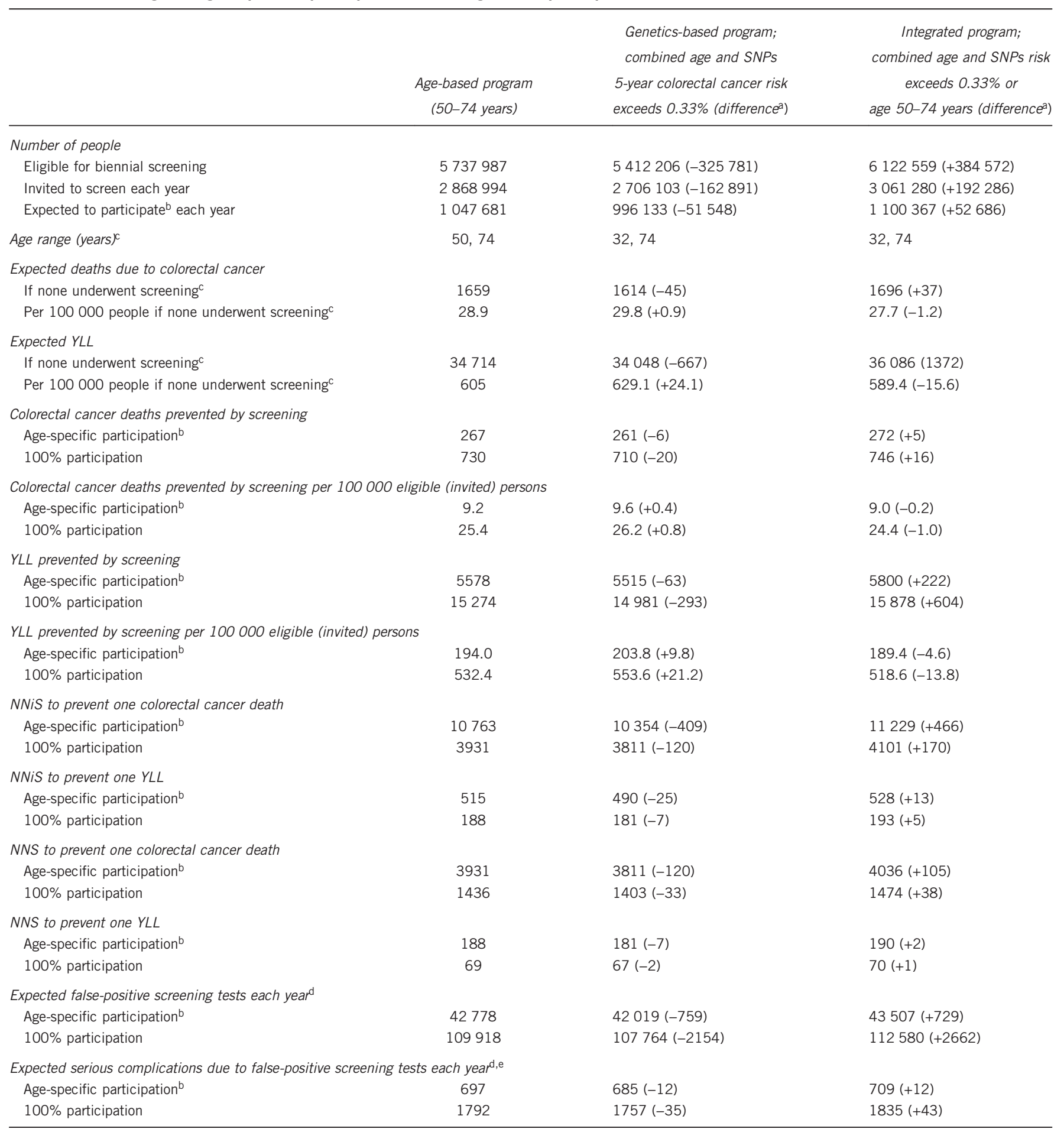

Abbreviations: NNiS, number needed to invite to screen; NNS, number needed to participate in screening.

aCompared with the age-based screening program.

${ }^{b}$ Age-specific participation rates, $\leq 54$ years $=0.274,55-59$ years $=0.359,60-64$ years $=0.427, \geq 65$ years $=0.406 .{ }^{20}$

In the biennially eligible population in 1 year.

${ }^{\mathrm{d}}$ Assuming positive predictive value of immunochemical fecal occult blood test (iFOBT) $=3.6 \% .{ }^{16}$

eAssuming $100 \%$ colonoscopy follow-up rate, and $1.6 \%$ of colonoscopy procedures result in an unplanned hospital visit due to serious complications (including hemorrhage, abdominal pain and perforation). 17 


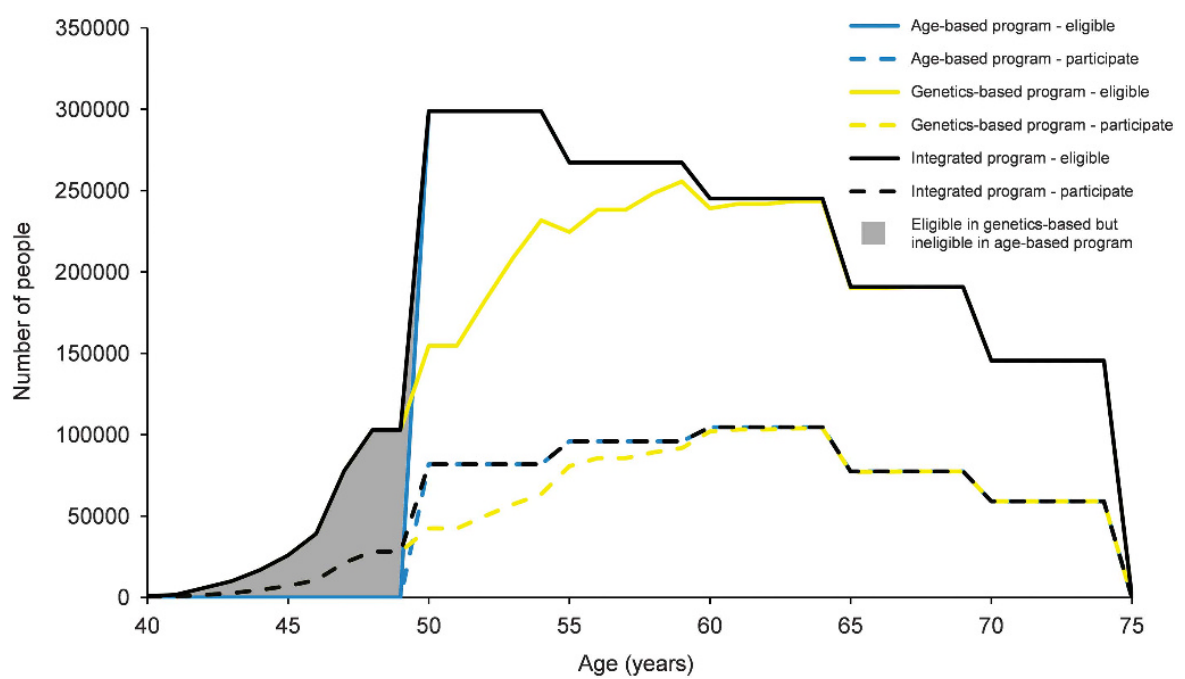

Figure 1 Histogram of number of people eligible (invited), and number of people who participate ${ }^{a b}$, in one year of biennial iFOBT screening according to age (years) in the hypothetical population $(N=22340$ 042), under the three programs; age-based, genetics-based and combined age-based and genetics-based (integrated). The number of people eligible to screen under the genetics-based program, but not eligible under the age-based program ${ }^{c}(N=192286)$,

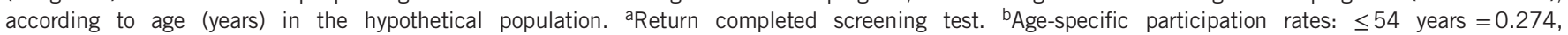
$55-59$ years $=0.359,60-64$ years $=0.427, \geq 65$ years $=0.406 .20$ People aged less than 50 years whose combined risk surpassed a $0.33 \% 5$-year risk of colorectal cancer (equivalent to the average population risk at age 50 years).

$(n=52686$, and all under 50) resulting in an overall $2.7 \%$ decrease in screening efficiency.

Directing screening to those at highest risk of colorectal cancer based on genetic risk for age rather than based on age alone would have the additional benefit of reduced false-positive screening tests. Further, by excluding people aged 50-74 years whose 5-year risk of colorectal cancer diagnosis is less than $0.33 \%$, the genetics-based program would avoid screening a proportion at relatively low risk of colorectal cancer death. Under the age-based program, the benefits (ie, deaths prevented and YLLs prevented) of screening this low risk segment of 50-74 year olds are relatively low, compared with others in the 50-74 years age-bracket. Although there are no direct physical adverse effects from iFOBT screening, ${ }^{2}$ false-positive screening test results may induce stress, anxiety and disruption to people's lives (albeit, the evidence of these effects is mixed ${ }^{18}$ ), and lead to unnecessary colonoscopy screening, ${ }^{2}$ which will result in a proportion having an adverse event due to the invasive nature of the test. We estimate that there would be approximately 18 events of serious complication due to colonoscopy of people who would be eligible under the age-based program, but not under the genetics-based program, and 12 fewer events overall under the genetics-based program compared with the age-based program each year. Although false-negative iFOBT screening test results are more deleterious than false-positive results, a screening program that limits false-positive iFOBT results is advantageous.

\section{Project limitations}

We assumed that a reduction in mortality rate of $44 \%$ could be achieved by biennial iFOBT screening if $100 \%$ of the eligible population participated in screening. This was based on an observed $22 \%$ mortality reduction in an existing screening program in which $50 \%$ participated in biennial iFOBT screening ${ }^{15}$ and assuming mortality rate reduction was relative to participation rate. Participation in the Australian NBCSP is comparatively low (36.0\%), therefore, we assumed screening produced a mortality rate reduction of $16 \%$ under incomplete participation.

This study's estimations rely on an availability of genetic information on 45 specific SNPs across the population. In addition to many important ethical and legal hurdles, attainment of genetic information requires a willingness of the population to participate. Although there is a strong public support for genetic testing for health purposes (for instance $89.1 \%$ of participants in a Dutch online panel survey of 55-59-year-olds reported they would take part in genetic screening for colorectal cancer $^{19}$ ), it is unknown how the offer of genetic testing for screening purposes may affect participation. Assuming eligibility under a genetics-based program would revert to being judged based on age risk for those who refuse to participate in genetic testing, a proportion of those eligible based on their genetic risk for age would, in reality, not be screened, and a proportion of those ineligible based on their genetic risk for age would, in reality, be screened. As genetic testing participation rates were not able to be accounted for, the estimated efficiency we report might be overstated.

For simplicity, we have not conducted SNP-specific risks. Rather we have simply used the averages of colorectal cancer risks for the number of risk alleles carried. Although the association with colorectal cancer risk for each allele might differ by SNP (and therefore for people with the same number of risk alleles, there will actually be a distribution of risk depending on which risk alleles were present), we do not expect this assumption to result in any biases in estimated efficiency.

In this study, a D prime $>0.5$ was deemed sufficient evidence that an SNP's association with colorectal cancer was due to linkage disequilibrium with another SNP in the same region. Application of a more-stringent threshold may have yielded fewer SNPs being included in the SNP risk model, thus decreasing the discriminatory accuracy for colorectal cancer and estimated efficiency of the geneticsbased screening programs.

We assumed that odds ratios associated with number of inherited SNPs do not vary according to age. The majority of studies that 
estimate relative risk according to SNPs have included commonly attainable cases ${ }^{7}-$ which, as colorectal cancer is a progressive disease, ${ }^{20}$ tend to be older individuals. By assuming that the risk of colorectal cancer diagnosis and death associated with SNPs does not vary according to age, we may have over- or underestimated the benefits of the genetics-based and integrated programs.

The frequency and associated risks (odds ratios) of the 45 included SNPs were estimated in populations of European descent. ${ }^{6}$ Estimated associated risks and population frequencies of numerous colorectal cancer-associated SNPs have varied according to ethnicity; ${ }^{21}$ as has risk of developing, and mortality from, colorectal cancer. ${ }^{22}$ Therefore, the results we have produced may not be applicable to non-Caucasian ethnicities.

One limitation of this study is that the age-specific estimates for 5 -year risk of being diagnosed with colorectal cancer do not account for the competing risk of death due to non-colorectal cancer causes. $^{23,24}$ Given that the rate of all-cause mortality increases with age, our method may disproportionately overestimate the 5-year risk of colorectal cancer diagnosis among older individuals who are at greater risk than younger people dying of non-colorectal cancer causes, and who therefore have less time to develop the disease, within the succeeding 5 years. ${ }^{23,24}$ Had competing risk of death from other causes been accounted for, there may have been a milder increase in the estimated age-specific risk of diagnosis between ages 70 and 75 years $^{25}$ than was observed in this study (Supplementary Figure 2). Therefore, this study may slightly overestimate the number of people (primarily older people) eligible to screen, and therefore the number of deaths and YYLs prevented, by the genetics-based screening program, and by the integrated program (although by a smaller margin for the latter given the relatively low rate of all-cause mortality in those under the age of 50 years).

This study's analyses are not stratified by gender, and therefore gender differences in age-specific colorectal cancer risk and their effect on screening eligibility and program efficiency, including whether genetic risk estimation is better for men or for women, was not considered. However, given it is the total number of deaths rather than the number of male or female deaths that is important to public health, using incidence rates and mortality data for males and females combined was appropriate for estimating the deaths prevented by the screening programs in this study. Although, as age-specific colorectal cancer incidence rates vary by gender, ${ }^{3}$ population risk stratification via combined gender, age and genetic risk has potential to further enhance the efficiency of colorectal cancer screening programs.

\section{Implications and future research}

Adopting a genetics-based screening program could benefit colorectal cancer screening in two ways: (i) by identifying the portion of the population aged less than 50 years who would benefit, in terms of preventing colorectal cancer deaths and YLLs, by screening; and (ii) increased efficiency by decreasing the number of screens to prevent a death or YLL. However, comparisons of a genetics-based program with existing age-based programs by numbers of people screened, numbers of deaths prevented and numbers of YLLs prevented are only one aspect of many that need to be addressed before full consideration can be given. Although this is an important first step, benefits and harms, cost-effectiveness, health-care resources, public, professional and policy-maker engagement and education requirements, and the myriad ethical, legal and social implications ${ }^{11,26}$ must be extensively researched before a pre-screening genetic test for screening eligibility is considered for colorectal cancer. Increasing the number of SNPs associated with colorectal cancer risk beyond the 45 assessed in this study will increase the ability of genetic risk profiling to stratify the population, and therefore the efficiency of screening programs incorporating genetic testing. With increasingly large collaborative GWAS studies and more sophisticated statistical methods to analyze the existing GWAS data, an increase in colorectal cancer-associated SNPs is expected.

\section{CONCLUSION}

We found evidence that a colorectal cancer screening program where eligibility is based on genetic risk for age is slightly more efficient at reducing colorectal cancer mortality than a standard program where eligibility is based on age given the number and ages of people screened, and can identify a proportion of those under 50 who would benefit from biennial iFOBT screening. Further evaluations assessing the financial, ethical and practical feasibility of implementing a genetics-based or integrated screening program, including population SNP-profiling, are required for the assessment of SNP-profiling for improving colorectal cancer screening.

\section{CONFLICT OF INTEREST}

The authors declare no conflict of interest.

\section{ACKNOWLEDGEMENTS}

MJ is funded by the National Health and Medical Research Council of Australia. The study titles, study leaders and funding sources for data sets used in this manuscript are as follows: 'Quantifying the utility of single-nucleotide polymorphisms to guide colorectal cancer screening', led by MJ; 'Australian cancer incidence and mortality (ACIM) book: Colorectal cancer (also called bowel cancer)', compiled by the Australian Institute of Health and Welfare, with data collection funded by each Australian state or territory through the jurisdictional departments of health (or equivalent) and some non-government organizations. The work of OS on this project was primarily completed as part of his Master's course at the University of Melbourne.

1 Hewitson P, Glasziou P, Watson E, Towler B, Irwig L: Cochrane systematic review of colorectal cancer screening using the fecal occult blood test (hemoccult): an update. Am J Gastroenterol 2008; 103: 1541-1549.

2 Whitlock EP, Lin JS, Liles E, Beil TL, Fu R: Screening for colorectal cancer: a targeted, updated systematic review for the U.S. Preventive Services Task Force. Ann Intern Med 2008; 149: 638-658.

3 Australian Institute of Health and Welfare: Australian Cancer Incidence and Mortality (ACIM) Books: Colorectal Cancer (Also Called Bowel Cancer). Canberra, ACT, Australia: Australian Institute of Health and Welfare, 2015.

4 Schreuders EH, Ruco A, Rabeneck L et al: Colorectal cancer screening: a globa overview of existing programmes. Gut 2015; 64: 1637-1649.

5 Johns LE, Houlston RS: A systematic review and meta-analysis of familial colorectal cancer risk. Am J Gastroenterol 2001; 96: 2992-3003.

6 Jenkins MA, Makalic E, Dowty JG et al: Quantifying the utility of single nucleotide polymorphisms to guide colorectal cancer screening. Future Oncol 2016; 12: 503-513.

$7 \mathrm{Hsu} \mathrm{L}$, Jeon J, Brenner $\mathrm{H}$ et al: A model to determine colorectal cancer risk using common genetic susceptibility loci. Gastroenterology 2015; 148.7: 1330-1339.

8 Dunlop MG, Tenesa A, Farrington SM et al: Cumulative impact of common genetic variants and other risk factors on colorectal cancer risk in 42103 individuals. Gut 2013; 62: 871-881

9 Frampton M, Houlston RS: Modeling the prevention of colorectal cancer from the combined impact of host and behavioral risk factors. Genet Med 2016: 19: 314-321.

10 LaFramboise T: Single nucleotide polymorphism arrays: a decade of biological, computational and technological advances. Nucleic Acids Res 2009; 37: 4181-4193.

11 Radice P, Pharoah PDP, Peterlongo P: Personalized testing based on polygenic risk score is promising for more efficient population-based screening programs for common oncological diseases. Ann Oncol 2016; 27: 369-370.

12 Pashayan N, Duffy SW, Neal DE et al: Implications of polygenic risk-stratified screening for prostate cancer on overdiagnosis. Genet Med 2015; 17: 789-795.

13 Pashayan N, Duffy SW, Chowdhury S et al: Polygenic susceptibility to prostate and breast cancer: implications for personalised screening. Br J Cancer 2011; 104: 1656-1663.

14 Australian Government Actuary: Australian Life Tables 2010-2012. Parkes, ACT, Australia: Commonwealth of Australia, 2014. 
15 Zorzi M, Fedeli U, Schievano E et al: Impact on colorectal cancer mortality of screening programmes based on the faecal immunochemical test. Gut 2015; 64: 784-790.

16 Australian Institute of Health and Welfare: Analysis of Bowel Cancer Outcomes for the National Bowel Cancer Screening Program. Canberra, ACT, Australia: Australian Institute of Health and Welfare, 2014.

17 Ranasinghe I, Parzynski CS, Searfoss R et al: Differences in colonoscopy quality among facilities: development of a post-colonoscopy risk-standardized rate of unplanned hospital visits. Gastroenterology 2016; 150: 103-113.

18 Kirkoen B, Berstad $\mathrm{P}$, Botteri $\mathrm{E}$ et al: Do no harm: no psychological harm from colorectal cancer screening. Br J Cancer 2016; 114: 497-504.

19 Veldwijk J, Lambooij MS, Kallenberg FGJ et al: Preferences for genetic testing for colorectal cancer within a population-based screening program: a discrete choice experiment. Eur J Hum Genet 2016; 24: 361-366.

20 Australian Institute of Health and Welfare: National Bowel Cancer Screening Program Monitoring report 2013-14. Canberra, ACT, Australia: Australian Institute of Health and Welfare, 2015
$21 \mathrm{He}$ J, Wilkens LR, Strain DO et al: Generalizability and epidemiologic characterization of eleven colorectal cancer GWAS hits in multiple populations. Cancer Epidemiol Biomarkers Prev 2011; 20: 70-81.

22 Ryu SY, Crespi CM, Maxwell AE: Colorectal cancer among Koreans living in South Korea versus California: incidence, mortality, and screening rates. Ethn Health 2014; 19: 406-423.

23 Pharoah P, Mackay J: Absolute risk of breast cancer in women at increased risk: a more useful clinical measure than relative risk? Breast 1998; 7: 255-259.

24 Dupont WD, Plummer WD: Understanding the relationship between relative and absolute risk. Cancer 1996; 77: 2193-2199.

25 Butterworth AS, Higgins JPT, Pharoah P: Relative and absolute risk of colorectal cancer for individuals with a family history: a meta-analysis. Eur J Cancer 2006; 42: 216-227.

26 Burton H, Chowdhury S, Dent T, Hall A, Pashayan N, Pharoah P: Public health implications from COGS and potential for risk stratification and screening. Nat Genet 2013; 45: 349-351.

Supplementary Information accompanies this paper on European Journal of Human Genetics website (http://www.nature.com/ejhg) 